\title{
Resonant pair-exchange scattering and BCS-BEC crossover in a system composed of dispersive and heavy incipient bands: A Feshbach analogy
}

\author{
Kazunari Ochi, ${ }^{1}$ Hiroyuki Tajima $\odot,{ }^{1,2}$ Kei Iida $\odot,{ }^{1}$ and Hideo Aoki $\odot^{2,3}$ \\ ${ }^{1}$ Department of Mathematics and Physics, Kochi University, Kochi 780-8520, Japan \\ ${ }^{2}$ Department of Physics, The University of Tokyo, Hongo, Tokyo 113-0033, Japan \\ ${ }^{3}$ Electronics and Photonics Research Institute, National Institute of Advanced Industrial Science \\ and Technology (AIST), Tsukuba 305-8568, Japan
}

(Received 29 July 2021; revised 10 November 2021; accepted 2 December 2021; published 13 January 2022)

\begin{abstract}
We theoretically show that a two-band system with very different masses harbors a resonant pair scattering that leads to novel pairing properties, as highlighted by the Bardeen-Cooper-Schrieffer (BCS) to Bose-Einstein condensation (BEC) crossover. Most importantly, the interband pair-exchange coupling induces an effective intraband attraction in each band, enhancing the superfluidity/superconductivity. The effect, a kind of SuhlKondo mechanism, is specifically enhanced when the second band has a heavy mass and is incipient (lying close to, but just above, the chemical potential $\mu$ ), which we call a resonant pair scattering. By elucidating the dependence of the effective interactions and gap functions on $\mu$, we can draw an analogy between the resonant pair scattering and the Feshbach resonance.
\end{abstract}

DOI: 10.1103/PhysRevResearch.4.013032

\section{INTRODUCTION}

One of the central issues in superconductivity/ superfluidity is the crossover between the Bardeen-CooperSchrieffer (BCS) and Bose-Einstein condensation (BEC) regimes, or a crossover between weak- and strong-coupling regimes [1-4]. Another crucial interest in recent years is the multiband superconductors and superfluids, which harbor many specific interests. Indeed there has been an upsurge of interests in multiband and multiorbital effects on superconductivity in a wide variety of strongly correlated solid-state systems as exemplified by the iron pnictides, copper oxides, and heavy-fermion compounds [5-10]. Multispecies cold-atom systems have also been intensively studied for exploring a variety of phenomena. Now, an intriguing question we want to elaborate on in the present work is as follows: What if we combine these two subjects to consider a BCS-BEC crossover in multiband superconductors and superfluids? Indeed, in solid-state systems, the iron-based superconductor is inherently multiband, and some compounds in the material family are considered to be in a BCS-BEC crossover regime. In cold-atom systems, there exists, in addition to the magnetic Feshbach resonance, what is called the "orbital Feshbach resonance" when the atomic spieces (such as $\mathrm{Yb}$ ) have inert electron spins but multiple orbital states. This can be utilized to provide with open and closed channels to realize the unitarity-limit region

Published by the American Physical Society under the terms of the Creative Commons Attribution 4.0 International license. Further distribution of this work must maintain attribution to the author(s) and the published article's title, journal citation, and DOI. in the crossover. Multiband systems also give us greater opportunities in that there are several degrees of freedom to be engineered, such as the mass ratio and band offset between the bands, relative positions between the chemical potential, and the respective band edges, where we can play around with interband versus intraband interactions in considering superconductivity/superfluidity.

A specific point of interest in multiband superconductors is what is called the "incipient band" situations. Namely, in some of the iron-based superconductors, the hole band has its edge located close to, but slightly away from, the chemical potential, which is called "incipient" [11-18]. While the terminology "incipient" is often used in the community of the iron-based FeSe superconductor for the incipient $s_{ \pm}$pairing involving the hole band below $E_{\mathrm{F}}$, the concept of the incipient situation itself was originally introduced in a 2005 paper [19]. Namely, the physics is that the pair scattering mediated by spin fluctuations occurs between the main band and incipient band [20-23], and this can drastically enhance superconductivity, especially when the incipient band is flat, as was found in Ref. [19]. In such situations, the interband pair scattering, on top of the intraband ones, crucially determines the gap symmetry [24,25] (see Ref. [26] for a review).

A further feature in the iron-based superconductors is that a compound $\mathrm{Fe}_{1+y} \mathrm{Se}_{x} \mathrm{Te}_{1-x}$ realizes crossover from the weakcoupling BCS regime to the BEC condensation of tightly bound pairs when the iron content $y$ is varied [27-30]. With decreasing $y$, the hole pocket becomes shallower, which makes the ratio $\Delta / E_{\mathrm{F}}$ between the superconducting gap and Fermi energy monotonically increase up to 0.5 [31], which has been regarded as an indication for the BCS-BEC crossover. Another solid-state system that accommodates the BCS-BEC crossover is a hafnium compound $\mathrm{Li}_{x} \mathrm{HfNCl}$ tuned with an electric-double-layer structure [32]. At a low carrier density 
$(x=0.04)$, a pseudogap reminiscent of strong-pairing fluctuations in the BCS-BEC crossover was observed, with $\Delta / E_{\mathrm{F}}$ reaching 0.12 at $x=0.02$. From theoretical viewpoints, it was proposed that similar resonant phenomena can occur in nanostructures with complicated geometries [33] or in tight-binding band structures [34].

If we turn to cold-atom systems, on the other hand, the unitarity limit in the BCS-BEC crossover was intensively investigated for the usual single-orbital, single-species ultracold Fermi gases [35,36], where $\Delta / E_{\mathrm{F}} \simeq 0.4-0.5$ was reported [37-39]. In the usual cold-atom systems, typically ${ }^{6} \mathrm{Li}$ and ${ }^{40} \mathrm{~K}$ Fermi atomic gases, are characterized by the $s$-wave scattering length $a$ for the interatomic interaction, which absorbs the ultraviolet divergence arising from the singular contact-type interaction. The quantity $a$ can be controlled by an external magnetic field with the magnetic Feshbach resonance associated with the electron-spin degree of freedom with $S=1 / 2$ [40]. The BCS-BEC crossover is marked by a change of sign of $a$, which is physically quite natural since it associates the crossover with the formation of a bound state for a pair. Now a realization of the two-band BCS-BEC crossover was recently anticipated in $\mathrm{Yb}$ Fermi gases [41-45]. In the case of the ${ }^{173} \mathrm{Yb}$ atom with $S=0$, the system accommodates the orbital Feshbach resonance, which involves intrachannel and interchannel interactions in a two-channel system having different electron-orbital states, ${ }^{1} S_{0}$ and ${ }^{3} P_{0}$, and nuclear-spin states $[4,41,46,47]$. The corresponding Hamiltonian is similar to the two-band superconductivity model called the Suhl-Kondo [24,25]. Moreover, a bound-state formation due to the two-band nature was demonstrated in recent experiments [48].

The orbital Feshbach resonance can also be invoked for inducing the Kondo effect by manipulating the spin exchange interaction in a two-band system $[49,50]$. Since the different orbital states of ${ }^{173} \mathrm{Yb}$ feel different optical-lattice potentials, this can be used to realize a two-band system having different effective masses. As we shall show, a kind of BCS-BEC crossover occurs in such a system, but that is driven by interband coupling and hence totally different from the usual single-band BCS-BEC crossover, where the scattering length alone is the controllable parameter.

With the above background, the purpose of the present work is to explore specific features in the BCS-BEC crossover that arise when we have a fermion system (single-species, spin-1/2) that consists of a lighter-mass band (called Band 1 hereafter) and a heavier-mass band (Band 2). We focus on what will happen when we engineer the system by varying a band offset $E_{0}$ along with the position of the chemical potential $\mu$ on top of the mass ratio of the two bands. For the reason mentioned above and elaborated below, we are specifically interested in the situation when Band 2 is "incipient," i.e., close to, but detached from, the chemical potential, as schematically depicted in Fig. 1. The questions we ask ourselves are as follows: Can the unusual superconducting or superfluid states arise when the mass ratio is large in the presence of intraband and interband pairing interactions? The interband interaction gives rise to interband pair scattering (i.e., virtual pair-exchange processes across the two bands). In two-band systems the gap function has two components, and we solve the two-component gap equation where we focus

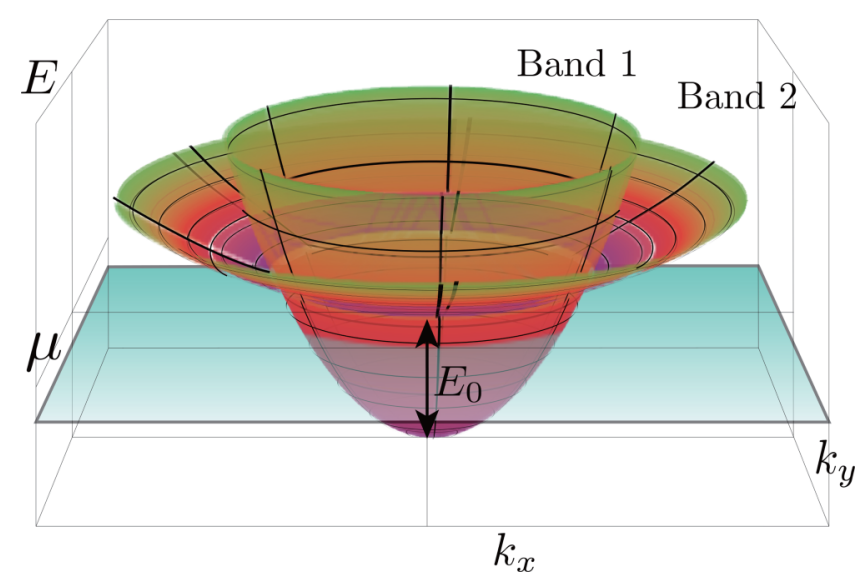

FIG. 1. Band dispersions [against $\left(k_{x}, k_{y}\right)$ with a $k_{z}=0$ projection in this plot] of the two-band system considered here with different effective masses with a band offset $E_{0}$. The upper band (Band 2) is assumed to have a heavier mass than the lower band (Band 1). Since we are interested in the situation where Band 2 is incipient (see text), the chemical potential $\mu$ is tuned around the bottom of Band 2.

on the intraband pairing in the case in which the chemical potential is set around the bottom of the incipient band.

We shall particularly clarify how the superfluid/ superconducting gaps and number densities behave in the presence of the resonant pair scattering by varying the mass ratio between the two bands. There, a point of interest is the effective scattering length [51-53] that characterizes the effective intraband interaction induced by the interband pair scattering. We shall show that the superfluid/superconducting gaps in the two bands are strongly enhanced in a manner drastically dependent on the band. This originates from the interband pair scattering when the incipient band is heavy, where the effective scattering lengths cross from the weak-coupling regime over to the strong-coupling one in a manner drastically dependent on the band.

This paper is organized as follows. In Sec. II, we present the two-band model Hamiltonian and formulate the gap equation to be solved numerically. We employ the mean-field BCS-Leggett theory $[1,4,54]$, which is known to successfully describe qualitative features of the BCS-BEC crossover at zero temperature in dilute systems as exemplified by cold atoms. While the BCS theory basically assumes that the excitation is restricted around the Fermi energy with the density of states taken as a constant, the BCS-Leggett theory employed in this paper includes excitations at shorter wavelengths. Such a difference is crucial for describing the BCS-BEC crossover in the that high-momentum excitations also occur in the strong-coupling regime where the Fermi surface is absent [4]. This is not directly applicable to the above-mentioned strongly correlated solid-state systems, but is expected to give a hint for the BCS-BEC crossover in multiband superconductivity.

Within the mean-field theory, the number density and the effective scattering length in each band are calculated. In Sec. III, we show numerical results for the chemical potential dependence of the superfluid/superconducting gaps, number density ratio, and effective scattering lengths. Section IV sum- 
(a)

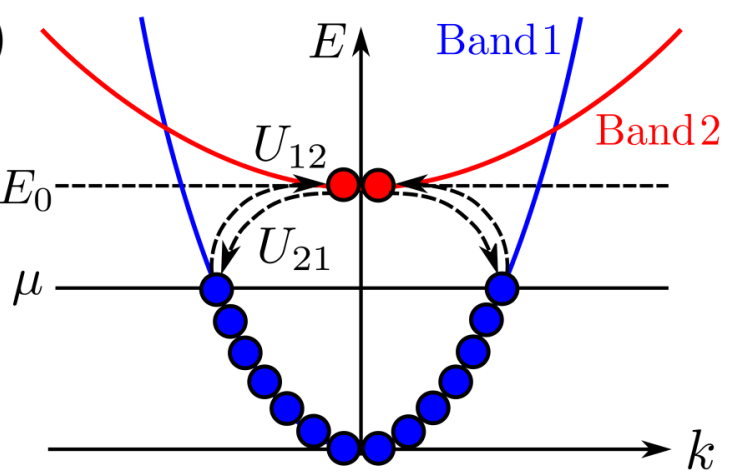

(b)
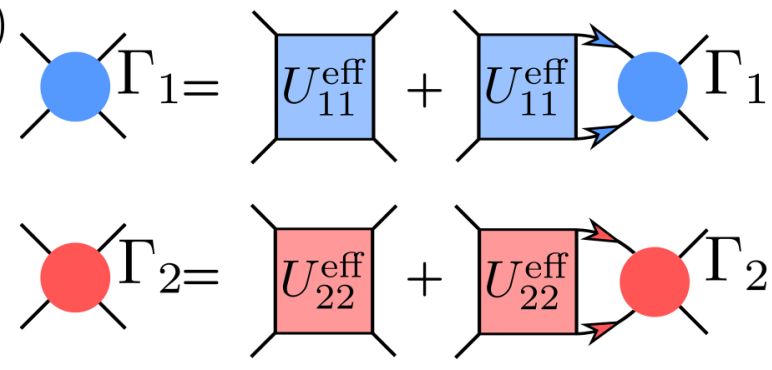

(c)
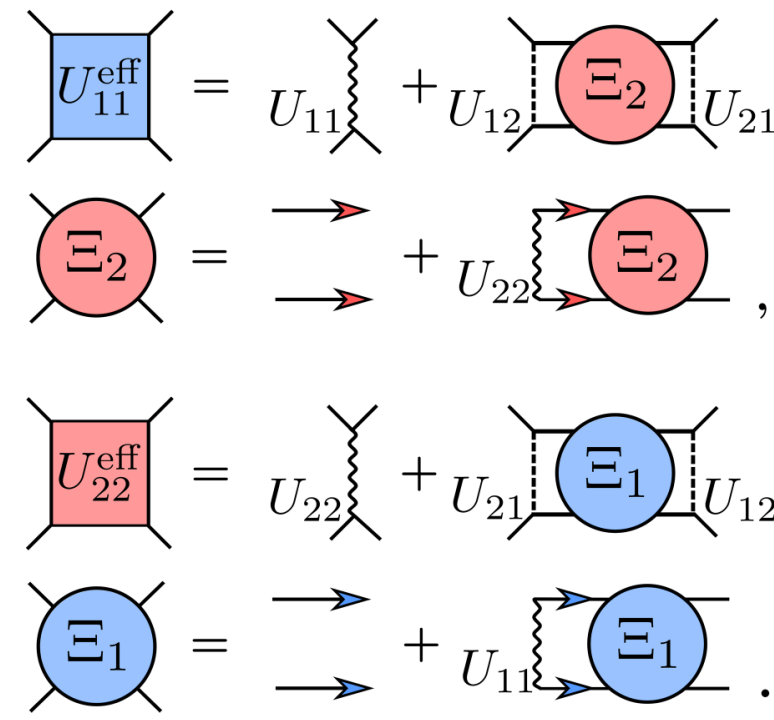

FIG. 2. (a) Interband pair-scattering processes are schematically shown on the band dispersion, here for $\mu<E_{0}$ in the presence of the pair-exchange interaction $U_{12}, U_{21}$. (b) Corresponding diagrams for the interaction vertex $\Gamma_{i}$ for Band $i$ [Eq. (9)], which is related with the effective scattering lengths $a_{i}^{\text {eff }}$ as defined in Eq. (9). (c) The effective intraband interactions $U_{i i}^{\text {eff }}$, Eq. (11), are composed of the bare intraband interaction ( $U_{i i}$; wavy lines) and the pair-exchange interaction between Band 1 and Band 2, which involves multiple scattering $\Xi_{j}$ in Band $j(\neq i)$.

marizes the paper. Throughout the text, we use units in which $\hbar=k_{\mathrm{B}}=1$, while the system volume is taken to be unity.

\section{FORMULATION}

As depicted in Fig. 1, we consider a two-band continuum model in three spatial dimensions, where the bands, with different masses and a band offset, have the dispersions

$$
\xi_{i}(\boldsymbol{k})=\frac{k^{2}}{2 m_{i}}-\mu+E_{0} \delta_{i, 2} .
$$

We assume a parabolic dispersion $\xi_{i}(\boldsymbol{k})$ against momentum $\boldsymbol{k}$ for each band labeled by the index $i=1,2$ having a mass (i.e., an effective mass for a lattice) $m_{i}, E_{0}$ is the band offset, and $\mu$ is the chemical potential. For a given value of $E_{0}$, we regard the chemical potential as a control parameter. The Hamiltonian reads [55]

$$
\begin{gathered}
H=\sum_{i, \boldsymbol{k}, \sigma} \xi_{i}(\boldsymbol{k}) c_{\boldsymbol{k}, \sigma, i}^{\dagger} c_{\boldsymbol{k}, \sigma, i}+\sum_{i, j} \sum_{\boldsymbol{k}, \boldsymbol{k}^{\prime}} V_{i j}\left(\boldsymbol{k}, \boldsymbol{k}^{\prime}\right) B_{\boldsymbol{k}, i}^{\dagger} B_{\boldsymbol{k}^{\prime}, j}, \\
B_{\boldsymbol{k}, j}=c_{-\boldsymbol{k}, \downarrow, j} c_{\boldsymbol{k}, \uparrow, j},
\end{gathered}
$$

where $c_{\boldsymbol{k}, \sigma, i}^{\dagger}$ creates a fermion with momentum $\boldsymbol{k}$ and spin $\sigma(=\uparrow$ or $\downarrow)$ in band $i$, and $B_{k, i}^{\dagger}$ is the pair-creation operator in band $i$. The second term in $H$ describes intraband $(i=j)$ and interband $(i \neq j)$ interactions. For the interaction $V_{i j}$, we assume in this paper, with cold-atom systems in mind, the contact-type attractive interaction

$$
V_{i j}\left(\boldsymbol{k}, \boldsymbol{k}^{\prime}\right)=-U_{i j} \theta(\Lambda-k) \theta\left(\Lambda-k^{\prime}\right),
$$

where $U_{i j} \geqslant 0$ and $\Lambda$ is a (spherical) momentum cutoff, which is required to avoid an ultraviolet divergence due to the contact-type interaction [4].

To renormalize the intraband interaction $U_{i i}$ against $\Lambda$, we can define, as a measure of $U_{i i}$, an $s$-wave intraband scattering length $a_{i}$ in Band $i$ as [4]

$$
\frac{4 \pi a_{i}}{m_{i}}=\frac{-U_{i i}}{1-U_{i i} \sum_{k}^{k \leqslant \Lambda} \frac{1}{k^{2} / m_{i}+2 E_{0} \delta_{i 2}}} .
$$

We apply the mean-field approximation to both the intra and interband pair-scattering processes in the Hamiltonian Eq. (2) for describing the superfluid/superconducting properties. The gap equation in a two-band system can be expressed in such a way that the two superfluid/superconducting gaps $\Delta_{1}, \Delta_{2}$ are coupled as $[56,57]$

$$
\Delta_{i}=\sum_{j=1,2} U_{i j} \Delta_{j} \sum_{k}^{k \leqslant \Lambda} \frac{\tanh \left(\frac{E_{j}(\boldsymbol{k})}{2 T}\right)}{2 E_{j}(\boldsymbol{k})},
$$

where $E_{j}(\boldsymbol{k})=\left[\xi_{j}^{2}(\boldsymbol{k})+\Delta_{j}^{2}\right]^{1 / 2}$ is the quasiparticle dispersion in the superfluid/superconducting state. For applying the mean-field approximation, the effect of inter-band pairscattering processes is nonperturbatively included in our two-band gap equation (5). This equation reproduces the twobody bound-state equation in the large interband-coupling limit (see Appendix A). In the limit where the interband interactions $U_{12}$ and $U_{21}$ are larger than the intraband interactions $U_{11}, U_{22}$, Eq. (5) corresponds to the gap equation in Eq. (25) of Ref. [58] where the interband pair scattering is dominant.

We note that Eq. (5) can also be obtained from the condition for the gapless collective mode in the $T$-matrix approximation [4]. Although its form is different from more sophisticated approaches such as the self-consistent $T$-matrix approximation, we employ the present formalism since the 
$T$-matrix approach based on Eq. (5) is successfully applied to the BCS-BEC crossover [59-61].

Since we are interested in the incipient situation, we tune $\mu$ around $\mu=E_{0}$ where $\mu$ touches the bottom of Band 2, in which the occupied number density $n_{i}$ in Band $i$ changes with $\mu$ as

$$
n_{i}=2 \sum_{\boldsymbol{k}}\left\{v_{i}^{2}(\boldsymbol{k}) f\left[-E_{i}(\boldsymbol{k})\right]+u_{i}^{2}(\boldsymbol{k}) f\left[E_{i}(\boldsymbol{k})\right]\right\},
$$

where $f\left[ \pm E_{i}(\boldsymbol{k})\right]=1 /\left(e^{ \pm E_{i}(\boldsymbol{k}) / T}+1\right)$ is the Fermi-Dirac distribution function, while the BCS coefficients are given as

$$
\begin{aligned}
& v_{i}^{2}(\boldsymbol{k})=\frac{1}{2}\left[1-\frac{\xi_{i}(\boldsymbol{k})}{E_{i}(\boldsymbol{k})}\right], \\
& u_{i}^{2}(\boldsymbol{k})=1-v_{i}^{2}(\boldsymbol{k}) .
\end{aligned}
$$

In the presence of the interband interaction $U_{12}$, we have the resonant pair-scattering, as shown in Fig. 2(a) and captured diagramatically in Fig. 2(b). We can then calculate the effective scattering length $a_{i}^{\text {eff }}$, which reflects the pair-exchange-induced intraband attraction in Fig. 2(b) as

$$
\frac{4 \pi a_{i}^{\text {eff }}}{m_{i}} \equiv \Gamma_{i}=\frac{-U_{i i}^{\text {eff }}}{1-U_{i i}^{\text {eff }} \sum_{k}^{k \leqslant \Lambda} \frac{1}{k^{2} / m_{i}+2 E_{0} \delta_{i 2}}} .
$$

Here $\Gamma_{i}$ is the interaction vertex, and $U_{i i}^{\text {eff }}$ is the effective interaction in Band $i$ that can be obtained by rewriting Eq. (5) as

$$
\Delta_{i}=U_{i i}^{\mathrm{eff}} \sum_{k}^{k \leqslant \Lambda} \frac{\Delta_{i}}{2 E_{i}(\boldsymbol{k})} \tanh \left(\frac{E_{i}(\boldsymbol{k})}{2 T}\right)
$$

with

$$
\begin{gathered}
U_{i i}^{\mathrm{eff}}=U_{i i}+U_{i j} \Xi_{j} U_{j i}, \\
\Xi_{j}=\frac{\sum_{k}^{k \leqslant \Lambda} \frac{\tanh \left(\frac{E_{j}\left(k^{\prime}\right)}{2 T}\right)}{2 E_{j}(\boldsymbol{k})}}{1-U_{j j} \sum_{k}^{k \leqslant \Lambda} \frac{\tanh \left(\frac{E_{j}\left(k^{\prime}\right)}{2 T}\right)}{2 E_{j}(k)}}
\end{gathered}
$$

for $(i, j)=(1,2)$ or $(2,1)$.

The BCS-BEC crossover is characterized in terms of the dimensionless coupling parameter, $1 /\left(k_{0} a_{i}\right)$, as [4]

$$
\begin{aligned}
& 1 /\left(k_{0} a_{i}\right) \rightarrow-\infty: \text { weak-coupling BCS limit, } \\
& 1 /\left(k_{0} a_{i}\right) \rightarrow+\infty: \text { strong-coupling BEC limit, }
\end{aligned}
$$

where $k_{0} \equiv \sqrt{2 m_{1} E_{0}}$ is the Fermi momentum as defined for a zero-temperature ideal Fermi gas having a mass $m_{1}$ and Fermi energy $E_{0}$ of Band 1 when Band 2 starts to be occupied. Since we want to focus on the effects of the pair-exchange coupling, the intraband couplings are taken to be weak as $1 /\left(k_{0} a_{1}\right)=1 /\left(k_{0} a_{2}\right)=-2$ throughout the present paper. The crossover of interest here is driven by interband coupling, hence it is distinct from the usual single-band crossover. We now examine how the $1 /\left(k_{0} a_{i}^{\text {eff }}\right)$ changes across the BCS to BEC regimes as $\mu$ is increased for various values of $\tilde{U}_{12}$ and $m_{1} / m_{2}$. The momentum cutoff is here taken to be $\Lambda=100 k_{0}$.
We numerically checked that the result does not change significantly for larger cutoffs.

\section{RESULTS AND DISCUSSIONS}

\section{A. Superfluid/superconducting gaps and particle densities}

The result for the gap functions against the chemical potential $\mu / E_{0}$, calculated from the mean-field Eq. (5), is displayed in Fig. 3 for $\Delta_{1}$ and Fig. 4 for $\Delta_{2}$ for the mass ratio $m_{1} / m_{2}=$ $1,0.25,0.1$. It is convenient to introduce a dimensionless interband pair-exchange coupling $[58,61]$

$$
\tilde{U}_{12} \equiv\left(\frac{\Lambda}{k_{0}}\right)^{2} \frac{n}{E_{0}} U_{12},
$$

where $n=k_{0}^{3} /\left(3 \pi^{2}\right)$ is the total particle density as defined for a zero-temperature ideal Fermi gas having a mass $m_{1}$ and a Fermi energy $E_{0}$. For each value of $m_{1} / m_{2}$ we vary the interband interaction $\tilde{U}_{12}$ from 0.0 to 2.0. The result for the inverse effective scattering length $1 / a_{i}^{\text {eff }}$, which serves as a measure of the interaction strength, is also shown in the lower panels of each figure.

We can see that both $\Delta_{1}$ and $\Delta_{2}$ increase with $\mu$, but in a way vastly dependent on $m_{1} / m_{2}$ and $\tilde{U}_{12}$, both in their magnitude and the functional form against $\mu$. The enhancement of $\Delta_{1}, \Delta_{2}$ by the presence of the interband pair-exchange coupling $U_{12}$ can be regarded as a Suhl-Kondo mechanism $[24,25]$, but, crucially, this occurs more intensively with orders of magnitude difference between $\Delta_{1}, \Delta_{2}$ for larger mass difference (i.e., smaller mass ratio $m_{1} / m_{2}$ ), as is typically seen in the result for $m_{1} / m_{2}=0.1$ where the incipient band dispersion becomes almost flat.

If we look at the band dependence more closely, $\Delta_{1}$ is always nonzero, while $\Delta_{2}$ vanishes for $\mu<E_{0}$ when $U_{12}=0$, which is because Band 2 is unoccupied as depicted in the result for the band occupancies in Fig. 5 which shows that the number density ratio $n_{2} / n_{1}$ is virtually zero for $\mu<E_{0}$ in the absence of $U_{12}$ regardless of the value of $m_{1} / m_{2}$. To be precise, even at $U_{12}=0$, the onset of nonzero density in Band 2 is slightly shifted toward the lower chemical potential with decreasing $m_{1} / m_{2}$, a feature due to the intraband attraction.

In the presence of $U_{12}$, on the other hand, $\Delta_{2}$ also becomes finite even for $\mu<E_{0}$. There, $\Delta_{1}$ and $\Delta_{2}$ become simultaneously finite through the coupling in Eq. (5) due to virtual pair-exchange processes. Band 2 occupancy $n_{2}$ also becomes significantly finite for $\mu<E_{0}$ due to $U_{12}$, implying the acquisition of pair condensation in the incipient band located above $\mu$.

Another characteristic feature is that both $\Delta_{1}$ and $\Delta_{2}$ remain finite even at $\mu=0$ when the mass ratio is small and the pair-exchange coupling is sufficiently large. Although this may seem strange, a bound state prevails in such a case as suggested in the context of a two-body problem. In this regime, the pair formation originates from the two-body bound state formation (as seen from the pole of the $T$-matrix discussed in Appendix A) rather than the Cooper instability. Indeed, we obtain finite two-body binding energies $E_{\text {bind }}$ at $\tilde{U}_{12}=1.5$ and 2.0 therein. The finite binding energy in the two-body problem is related to positive values of $1 /\left(k_{0} a_{1}^{\text {eff }}\right)$ 

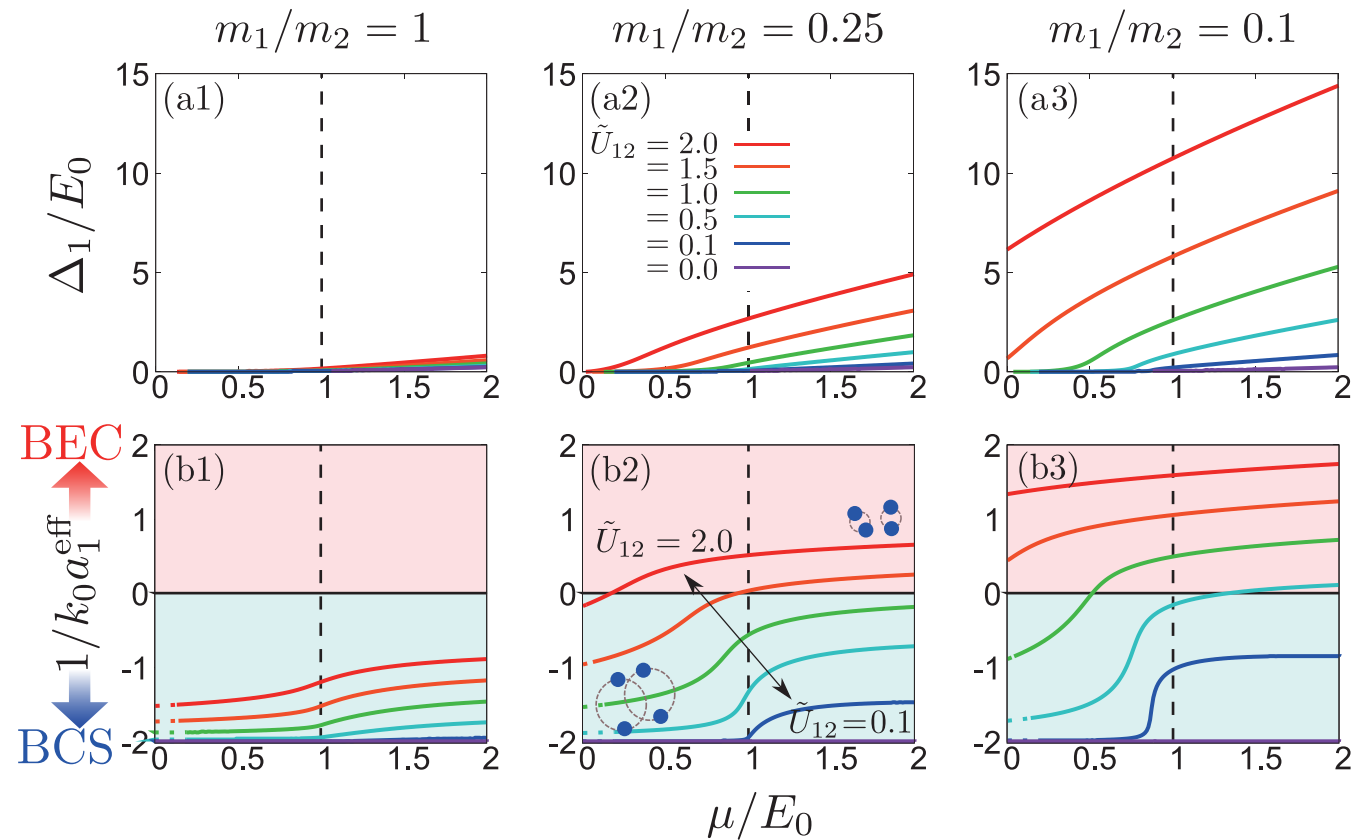

FIG. 3. Superfluid/superconoducting gap $\Delta_{1}$ (upper panels) and the inverse effective scattering length $1 / a_{1}^{\text {eff }}$ (lower) in Band 1 calculated as functions of the chemical potential $\mu$ at different mass ratios $m_{1} / m_{2}=1$ (left panels), $m_{1} / m_{2}=0.25$ (middle), and $m_{1} / m_{2}=0.1$ (right). In each panel the result is obtained for various values of the pair exchange coupling $\tilde{U}_{12}=0.0,0.1,0.5,1.0,1.5$, and 2.0 as color coded. We take $1 /\left(k_{0} a_{1}\right)=1 /\left(k_{0} a_{2}\right)=-2$. At $\tilde{U}_{12}=0.0,1 /\left(k_{0} a_{1}^{\text {eff }}\right)$ coincides with $1 /\left(k_{0} a_{1}\right)=-2$. The horizontal solid lines at $1 /\left(k_{0} a_{1}^{\text {eff }}\right)=0$ represent the unitarity limit, while the vertical dashed lines mark $\mu=E_{0}$. For $\mu \rightarrow 0$ where $\Delta_{2}$ is negligibly smaller than $E_{0}$, we display the asymptotic solutions obtained from the two-body calculation at $\mu=0$ (see Appendix A) as dotted curves.

at $\mu=0$ in Fig. 3(b3). However, we should note that this argument does not hold for Band 2 because $1 /\left(k_{0} a_{2}^{\text {eff }}\right)$ for $\mu \rightarrow 0$ deviates from the result of Lippmann-Schwinger equation due to the many-body effect as discussed in Eq. (17) below, i.e., $\Delta_{1}$ exerts a significant effect in Eq. (12) for $(i, j)=(2,1)$. While $n_{2}$ increases with $m_{2}$ largely due to the increased density of states, the interband pair-exchange acts to reduce $n_{2} / n_{1}$ above $\mu=E_{0}$ as a result of the enhanced effective intraband attraction in Band 1, which we shall discuss below.
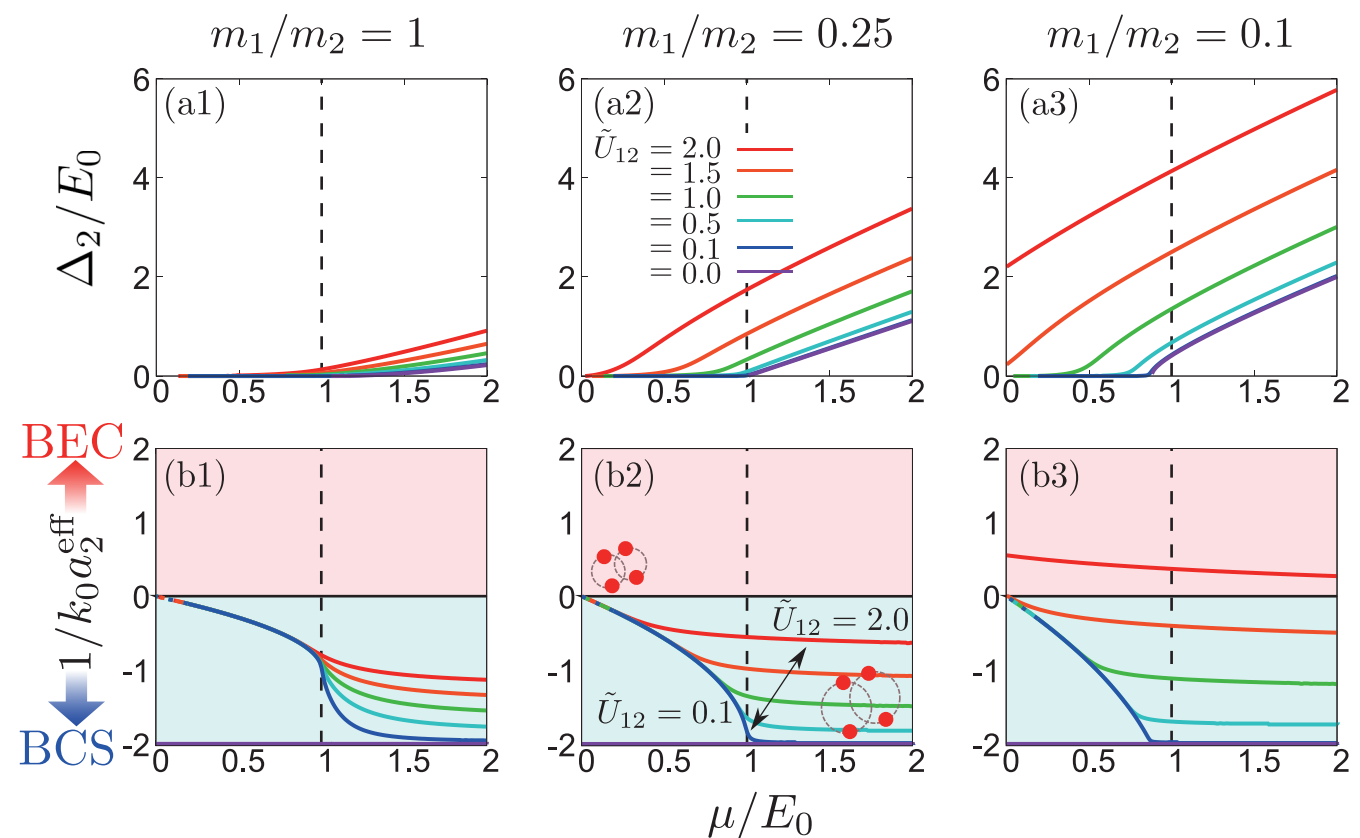

FIG. 4. Same as Fig. 3 for Band 2. The behavior for $\mu \rightarrow 0$ with $1 /\left(k_{0} a_{2}^{\text {eff }}\right) \rightarrow 0$ is displayed as dotted lines following Eq. (17). 

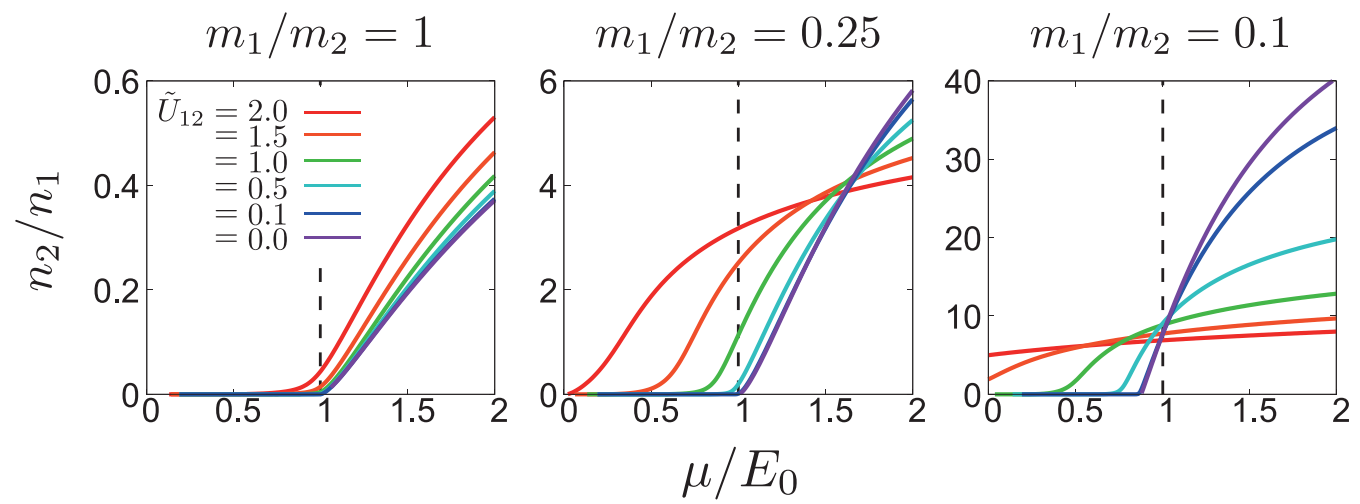

FIG. 5. Particle density ratio $n_{2} / n_{1}$ calculated for the mass ratio $m_{1} / m_{2}=1$ (left panel), $m_{1} / m_{2}=0.25$ (middle), and $m_{1} / m_{2}=0.1$ (right) for various values of the pair exchange coupling $\tilde{U}_{12}$. The vertical dashed lines mark $\mu=E_{0}$. The intraband scattering lengths are set to $1 /\left(k_{0} a_{1}\right)=1 /\left(k_{0} a_{2}\right)=-2$ as in Figs. 3 and 4 .

\section{B. Effective scattering length in each band}

We revealed in Figs. 3 and 4(b1) to 4(b3) that the inverse effective scattering lengths, $1 /\left(k_{0} a_{1}^{\text {eff }}\right)$ and $1 /\left(k_{0} a_{2}^{\text {eff }}\right)$ defined in Eq. (9), have dramatically different dependence on the chemical potential when we vary the mass ratio $m_{1} / m_{2}$. Based on the result, we can now argue how the BCS-BEC crossover evolves with $\mu$ in the present two-band model for various values of the interactions $U_{i j}$. The situation is indeed in a sharp contrast with an ultracold Fermi gas around the magnetic Feshbach resonance where the BCS-BEC crossover can be realized by tuning the attractive interaction alone.

The effective intraband attraction in Band 1 as measured by $1 /\left(k_{0} a_{1}^{\text {eff }}\right)$ significantly and monotonically increases with $\mu$, where the value changes from negative to positive (i.e., $a_{1}^{\text {eff }}$ itself diverges) typically in Figs. 3(b2) and 3(b3) for smaller mass ratios. The sign change happens around $\mu=E_{0}$ where $\mu$ touches the bottom of the incipient band. We can capture its mechanism as schematically depicted in Fig. 6. There, we compare the pair scattering processes in the present two-band model with the conventional magnetic Feshbach resonance in an ultracold single-atomic-species Fermi gas. In the Fermi gas, the effective scattering length $a_{\text {eff }}^{\text {Fesbach }}$ between atoms in the two-channel model is given by [4]

$$
\frac{4 \pi a_{\mathrm{eff}}^{\text {Feshbach }}}{m_{\mathrm{A}}}=U_{\mathrm{bg}}-\frac{g^{2}}{v} \frac{1}{1-\left(2 \mu_{\mathrm{A}} / v\right)},
$$

where $m_{\mathrm{A}}$ is the atomic mass, $\mu_{\mathrm{A}}$ the chemical potential, and $U_{\text {bg }}$ the background interaction. The attraction is induced by the Feshbach coupling $g$ between open-channel atoms and closed-channel molecules that have an energy level at $\nu$. One can see in the above equation that $a_{\text {eff }}^{\text {Feshach }}$ diverges at $\mu_{A}=$ $v / 2$ due to the resonance tuned by $\mu_{A}$. In the present two-band system, $a_{1}^{\text {eff }}$ diverges and changes sign (with $a_{2}^{\text {eff }}$ also rapidly changing; see Fig. 4) around $\mu=E_{0}$ for small $\tilde{U}_{12}$. So we can regard this, where the resonant pair scattering arises, as an analog of the Feshbach resonance accompanying a divergent $a_{\mathrm{eff}}^{\text {Feshbach }}$. To be more precise, the change of $1 / a_{1}^{\text {eff }}$ is related to the fact that $\Delta_{2}$ starts to increase around $\mu=E_{0}$ when $U_{12}$ is small (see Appendix B). Thus there exists an analogy between the two-band system and the conventional Feshbach resonance, although there are some differences between the two models (such as the Feshbach resonance being described by the coupling between the continuum and a bound state, whereas the resonant mechanism in the present two-band system originating from the coupling between two continua). In this analogy, $U_{12}$ in the two-band model plays the role of $g$ in the magnetic Feshbach resonance. So we can summarize the analogy as in Table I.

As exhibited conceptually in Fig. 6(a) and numerically in Fig. 3(b3) for $\tilde{U}_{12}=2,1 /\left(k_{0} a_{1}^{\text {eff }}\right)$ becomes large in a wide region of $\mu$ in contrast to the weak pair-exchange case when $U_{12}$ is large and $m_{1} / m_{2}$ is small. Such a situation corresponds, in the present analogy, to the so-called "broad Feshbach res-
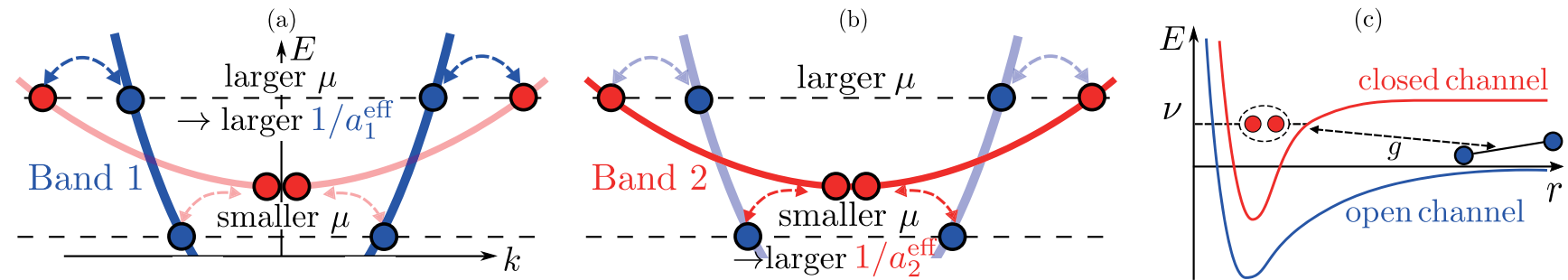

FIG. 6. Conceptual correspondence between (a,b) the present two-band system and (c) the two-channel model conventionally used for the Feshbach resonance. In (a) for Band 1, a virtual pair scattering from around the Fermi energy in Band 1 to Band 2 is depicted in the momentum space. Panel (b) depicts the pair scattering from Band 2 to Band 1. In panel (c) the process of bound-state formation is depicted for the closed and open channels against the relative coordinate $(r)$ of two atoms, where $g$ is the Feshbach coupling, and $v$ is the energy level of the closed channel. Then Band 2 can be analogous to a closed channel, although Band 2 does not always form a bound state. 
Table I. Relationship between the present two-band model for resonant pair-exchange scattering and the atomic two-channel model for the Feshbach resonance.

\begin{tabular}{lcc}
\hline \hline & Present two-band & Feshbach resonance \\
\hline Resonance energy & $\mu \simeq E_{0}$ & $\mu_{A}=v / 2$ \\
Coupling & $U_{12}$ & $g$ \\
\hline \hline
\end{tabular}

onance" as illustrated in Fig. 6 in that $1 /\left(k_{0} a_{1}^{\text {eff }}\right)$ is strongly enhanced as the interband interaction increases over a broad range of $\mu$ around $E_{0}$. In this way, the Band 1 crosses from the weak-coupling BCS regime over to the strong-coupling BEC regime with $\mu$ increasing across $E_{0}$ when $m_{1} / m_{2}$ is sufficiently small and $\tilde{U}_{12}$ sufficiently large. In particular, the effective interaction in Band 1 for large $U_{12}$ enters the strong-coupling regime even before $\mu$ reaches the bottom of Band 2 . Note that one of the important differences between the present two-band model and two-channel atomic systems is the fact that Band 1 in the two-band model cannot be reduced to a single-channel model due to the large density of states in Band 2, which results in the enhancement of $n_{2} / n_{1}$ in Fig. 5 for $\mu<E_{0}$. For $\mu \gtrsim E_{0}$, on the other hand, the strong effective interaction in Band 1 in that regime acts to enhance $n_{1}$, and hence reduces $n_{2} / n_{1}$ in Fig. 5 .

The enhanced pairing effect associated with analogy between the two-band model and two-channel atomic system occurs in both cases of the system coupled with bosonic and fermionic bands. We also note that in Ref. [34] a similar mechanism of the Feshbach resonance was proposed for a two-body problem in a two-channel tight-binding model with equal effective masses. There, a Feshbach resonance in the long-wavelength limit was discussed in terms of the scattering length and phase shift for varied one- and two-body potentials to reveal that the resonance can occur even when the closed channel has no bound states. The present study, by contrast, shows that the Feshbach analog arises driven by the chemical potential without changing any model parameters such as $U_{i j}$ and $E_{0}$. Also, we study here a many-body system, where a nontrivial realization of the unitarity limit in Band 2, in particular, is induced by the coupled two superconducting order parameters, which would be outside a two-body scattering.

If we turn to the incipient, heavy-mass Band 2, on the other hand, the effective intraband interaction within the incipient band reaches the unitarity limit, that is, $1 /\left(k_{0} a_{2}^{\text {eff }}\right) \rightarrow 0$ for $\mu \rightarrow 0$ in Fig. 4. We can also notice for the case of weak pair-exchange coupling that the $\mu$ dependence of $1 /\left(k_{0} a_{2}^{\text {eff }}\right)$ falls upon a universal behavior in the small $\mu$ limit for various values of $\tilde{U}_{12}$. This unitarity-limit behavior occurs as long as $\tilde{U}_{12}$ is nonzero (note that $a_{2}^{\text {eff }}=a_{2}$ for $\tilde{U}_{12}=0.0$ ). In fact, we can show in Appendix A that, whereas $1 /\left(k_{0} a_{1}^{\text {eff }}\right)$ coincides with the two-body calculation at $\mu \rightarrow 0$ regardless of the value of $\tilde{U}_{12}, 1 /\left(k_{0} a_{2}^{\text {eff }}\right)$ deviates significantly from the twobody calculation in the same limit in the presence of a nonzero $\tilde{U}_{12}$. This deviation stems from the coherent coupling between the binary condensates in the two-band system through the gap Eq. (5), from which we can rewrite $U_{22}^{\text {eff }}$ as

$$
U_{22}^{\text {eff }}=\frac{1}{\sum_{k}^{k \leqslant \Lambda} \frac{1}{2 E_{2}(k)}} .
$$

Note that the right-hand side of the above equation does not depend explicitly on $\tilde{U}_{12}$, a feature related to the aforementioned universal behavior of $1 /\left(k_{0} a_{2}^{\text {eff }}\right)$ for small $\mu$ and $\tilde{U}_{12}$. At $\mu \rightarrow 0$ and $\Delta_{2} / E_{0} \simeq 0$, Eq. (17) reduces to $U_{22}^{\text {eff }} \simeq$ $\left[\sum_{k}^{k \leqslant \Lambda} \frac{1}{k^{2} / m_{2}+2 E_{0}}\right]^{-1}$, leading to $1 / a_{2}^{\text {eff }} \rightarrow 0$ in Eq. (9). This nontrivial realization of a unitarity limit in the incipient band can also be interpreted as a narrow resonance mechanism as opposed to the broad resonance, where the "narrow" means that the change of the effective scattering length occurs in a narrow range of the tuning parameter $(\mu$ in the present model, a counterpart to $v$ in atomic systems); see more details in Appendix B. In other words, in the narrow resonance the interband interaction $(g)$ is weak, where the resonance occurs abruptly in the vicinity of the resonce condition. Thus we can give a picture of the broad resonance for Band 1 with strong interband interaction, and the narrow resonance for Band 2 with weak interband interaction. Incidentally, this situation does not apply when the bound states are formed for small $m_{1} / m_{2}$ and large $\tilde{U}_{12}$ as shown in Fig. 4(b3) $\left(\tilde{U}_{12}=2.0\right.$, red line), where the pairing is insensitive to the change of the chemical potential as compared with the case of the Cooper instability where the Fermi surface effect is crucial.

On the other hand, when $\tilde{U}_{12}$ is small, the $1 /\left(k_{0} a_{2}^{\text {eff }}\right)$ depends sensitively on the position of $\mu$ relative to $E_{0}$. The qualitative behavior of $1 /\left(k_{0} a_{2}^{\text {eff }}\right)$ around $\mu=E_{0}$ can again be understood by analogy with the Feshbach resonance. Namely, the light-mass band and the heavy-mass (incipient) band correspond, respectively, to the closed and open channels, as depicted in Fig. 6(b). In the context of the atomic two-channel model, assuming $v \rightarrow-|v|$, [which corresponds to treating Band 2 as the open channel in the two-channel model described by Eq. (16)], we obtain

$$
\frac{4 \pi a_{\mathrm{eff}}^{\text {Feshbach }}}{m_{\mathrm{A}}}=U_{\mathrm{bg}}+\frac{g^{2}}{|\nu|+2 \mu_{\mathrm{A}}},
$$

which indicates $4 \pi a_{\mathrm{eff}}^{\text {Feshbach }} / m_{\mathrm{A}} \rightarrow U_{\mathrm{bg}}$ for $\mu_{\mathrm{A}} \rightarrow \infty$. Correspondingly, by regarding the scattering continuum in Band 1 as the low-energy closed channel located at $-E_{0}$ below the Band 2 bottom, and by identifying $U_{\mathrm{bg}}$ with the bare intraband interaction in our two-band model, we can again establish a correspondence with the atomic model. This way, one can obtain the analogy in terms of the effective scattering lengths between the two-channel model and the Feshbach resonance in atomic systems. Indeed, despite various differences between the two models, $1 /\left(k_{0} a_{2}^{\text {eff }}\right)$ still approaches $1 /\left(k_{0} a_{2}\right)$ (taken to be -2 here) for $\mu \gtrsim E_{0}$, as shown in Figs. 4(b1) to 4(b3). In this regard, the incipient band crosses from the unitarity limit over to the weakly-coupling regime with increasing $\mu$, which is just opposite to Band 1 where $1 /\left(k_{0} a_{1}^{\text {eff }}\right)$ increases with $\mu$. When $\tilde{U}_{12}$ is large, Band 2 remains around the crossover even in the high-density regime $\left(\mu \gtrsim E_{0}\right)$.

\section{CONCLUSION}

In this paper, we investigated the effects of the resonant pair-exchange coupling and the resultant BCS-BEC and unitarity-BCS crossover in a two-band model consisting of dispersive and incipient nearly flat bands. Within the meanfield theory, we elucidate the chemical potential dependence 


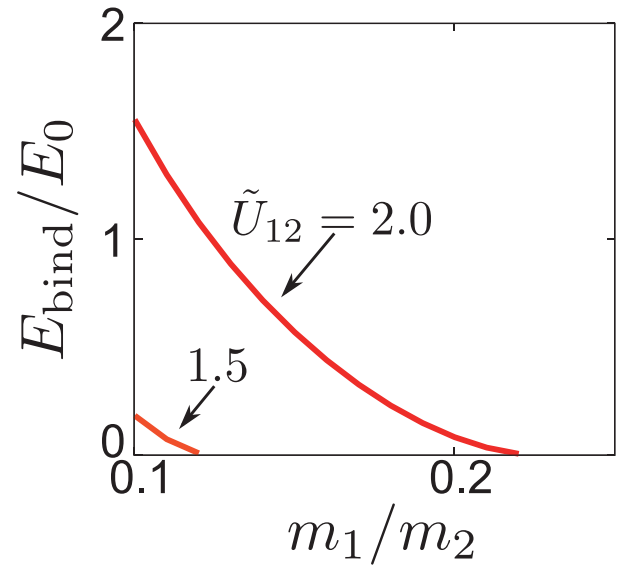

FIG. 7. The binding energy $E_{\text {bind }}$ calculated against $m_{1} / m_{2}$ for the $\tilde{U}_{12}=1.5$ and 2.0 .

of the superfluid/superconducting gaps and effective intraband interactions induced by the interband pair-exchange processes at various strengths of the pair-exchange coupling and effective mass ratio between the two bands. We found that superfluid/superconducting gaps in both bands are strongly enhanced when the incipient band becomes flatter. The effective scattering lengths which characterize the pairexchange-induced effective attraction in the dispersive band are tuned from the weak-coupling to strong-coupling regimes only by increasing the chemical potential, leading to the BCSBEC crossover without invoking any change in the coupling parameters. We discussed the analogy between the magnetic Feshbach resonance and the present two-band model in the presence of the incipient band. Moreover, the nontrivial realization of the unitarity limit in the incipient band was pointed out in the case of the small chemical potential, leading to the unitarity-BCS crossover with increasing $\mu$.

From an experimental point of view, while the effective scattering lengths cannot directly be measured in electronic systems, the BCS-BEC crossover can be observed by measuring energy spectra in tunneling spectroscopies (STM/STS), which should exhibit quite different behaviors between the $\mathrm{BCS}$ and BEC regimes. Moreover, angular-resolved photoe- mission spectra (ARPES) should give detailed information on quasiparticle spectra, as has actually been done for the iron-based superconductors for detecting a BCS-BEC crossover [31].

Although our model is rather simplified in describing real materials, such as iron-based superconductors and bilayer graphenes, our results would be useful for understanding the strong-coupling properties of multiband superfluid/superconductors. Moreover, our approach could be applied to the topological flat band system as well as lattice models. Thermal pairing fluctuations also play a crucial role throughout the BCS-BEC crossover. These remain as important future work.

\section{ACKNOWLEDGMENTS}

K.I. was supported by a Grant-in-Aid for scientific Research from JSPS (Grant No. 18H01211). K.I. and H.T. were supported by a Grant-in-Aid for scientific Research from JSPS (Grant No. 18H05406). H.A. thanks Core Research for Evolutional Science and Technology "Topology" project from the Japan Science and Technology Agency (Grant No. JPMJCR18T4), and JSPS KAKENHI (Grant JP17H06138).

\section{APPENDIX A: COMPARISON BETWEEN TWO-BODY AND MANY-BODY SCATTERING PROPERTIES}

Here, we summarize two-body properties in the present two-band system. For convenience, we define a $2 \times 2$ matrix $\hat{V}$ for the coupling constants in the band basis

$$
\hat{V} \doteq\left(\begin{array}{ll}
-U_{11} & -U_{12} \\
-U_{21} & -U_{22}
\end{array}\right)
$$

The in-vacuum two-body propagator is given by

$$
\hat{J}\left(\omega_{+}\right) \doteq\left(\begin{array}{cc}
J_{1}\left(\omega_{+}\right) & 0 \\
0 & J_{2}\left(\omega_{+}\right)
\end{array}\right),
$$

where $\omega_{+}$is the two-particle energy with an infinitesimal imaginary part $+\mathrm{i} \delta$ and

$$
J_{i}\left(\omega_{+}\right)=\sum_{k}^{k \leqslant \Lambda} \frac{1}{\omega_{+}-\left(k^{2} / m_{i}+2 E_{0} \delta_{i 2}\right)} .
$$
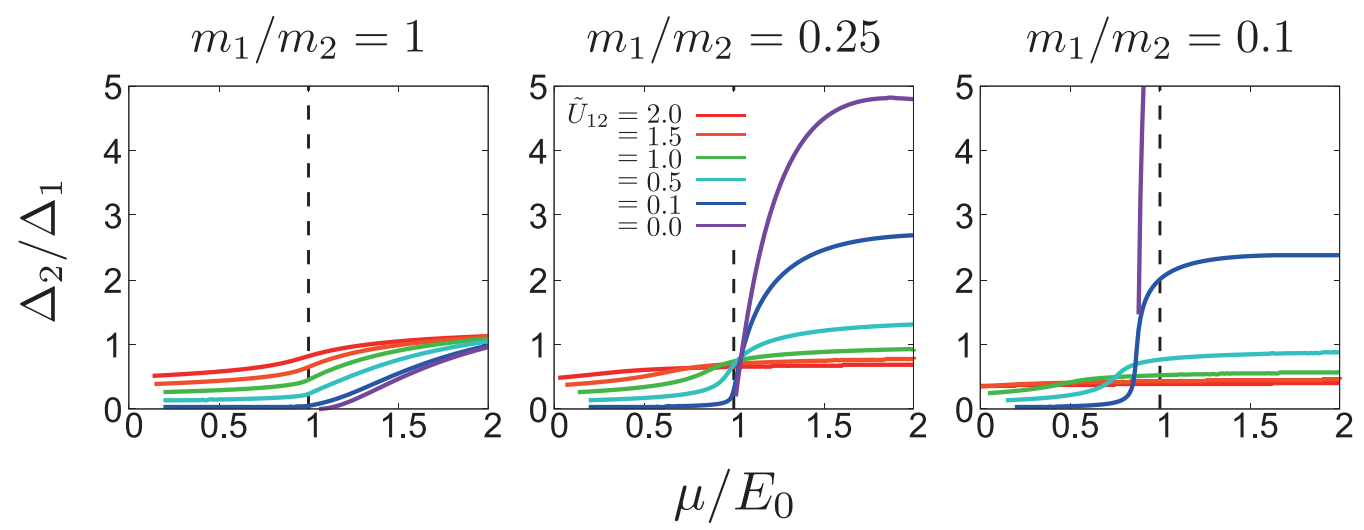

FIG. 8. The ratio $\Delta_{2} / \Delta_{1}$ of the gap functions as shown in Figs. 3(a1) to 3(a3), Figs. 4(a1) to 4(a3), is displayed for the mass ratio $m_{1} / m_{2}=1$ (left panel), $m_{1} / m_{2}=0.25$ (middle), and $m_{1} / m_{2}=0.1$ (right) for various values of the pair exchange coupling $\tilde{U}_{12}$. The vertical dashed lines mark $\mu=E_{0}$. 
We consider the diagonal component of the two-body $2 \times$ $2 T$-matrix element $T_{i}\left(\omega_{+}\right)$in Band $i$, which is given by

$$
T_{i}\left(\omega_{+}\right)=\frac{\bar{U}_{i i}^{\text {eff }}}{1-\bar{U}_{i i}^{\text {eff }} J_{i}\left(\omega_{+}\right)},
$$

where

$$
\bar{U}_{i i}^{\text {eff }}=U_{i i}+U_{i j} \frac{J_{j}\left(\omega_{+}\right)}{1-U_{j j} J_{j}\left(\omega_{+}\right)} U_{j i}
$$

is the two-body effective intraband interaction in Band $i$. In the strong-coupling regime, we can obtain the two-body binding enegy $-E_{\text {bind }}<0$ in Band 1 (which gives $-E_{\text {bind }}+2 E_{0}$ for the two-body binding energy in Band 2) from the pole of Eq. (A4) as

$$
1=\bar{U}_{i i}^{\text {eff }} J_{i}\left(-E_{\text {bind }}\right),
$$

as shown in Fig. 7. The presence of a nonzero $E_{\text {bind }}$ indicates that $\Delta_{1}, \Delta_{2}$ can be finite even at $\mu=0$ (as shown in Figs. 3 and 4$)$.

The low-energy limit $\omega_{+} \rightarrow 0$ of $\bar{U}_{11}^{\text {eff }}$ coincides with Eq. (11) in the main text for $i=1$ at $\mu \simeq 0$ since $\Delta_{2} / E_{0} \simeq 0$ even in the many-body counterpart. On the other hand, $U_{22}^{\text {eff }}$ does not coincide with $\bar{U}_{22}^{\text {eff }}$. More details about the deviation between $U_{22}^{\text {eff }}$ and $\bar{U}_{22}^{\text {eff }}$ are given in Appendix B, below.

We can further consider a situation in which the two-band system is in the BEC limit $\left(\mu<0, \Delta_{1}, \Delta_{2} \ll|\mu|\right)$ even when only the interband interaction exists with no intraband ones (large interband-coupling limit). Equation (10) rewritten from Eq. (5) is then approximated to

$$
\begin{aligned}
1 \simeq & U_{i j} \sum_{k}^{k \leqslant \Lambda} \frac{1}{k^{2} / m_{j}+2|\mu|+2 E_{0} \delta_{2 j}} U_{j i} \\
& \times \sum_{k}^{k \leqslant \Lambda} \frac{1}{k^{2} / m_{i}+2|\mu|+2 E_{0} \delta_{i 2}}
\end{aligned}
$$

in the BEC limit for $i \neq j$. The chemical potential in the BEC limit satisfies the same equation (A6) as that for the twobody binding energy in the absence of intraband interactions.
Therefore, we obtain

$$
\mu=-\frac{E_{\text {bind }}}{2} \text {. }
$$

This equation is similar to the single-band case, where the chemical potential asymptotically approaches the result for half the two-body binding energy in the BEC limit at zero temperature.

\section{APPENDIX B: LOW-DENSITY LIMIT OF $1 /\left(k_{0} a_{i}^{\text {eff }}\right)$}

Let us here clarify the mechanism by which $1 /\left(k_{0} a_{2}^{\text {eff }}\right)$ approach the unitarity limit despite the small pair-exchange interactions as long as $U_{12}$ is nonzero, while $1 /\left(k_{0} a_{1}^{\text {eff }}\right)$ is in the BCS regime for $\mu<E_{0}$.

First, note that $U_{i i}^{\text {eff }}$ can be cast into a form

$$
U_{i i}^{\text {eff }}=U_{i i}+U_{i j} \frac{\Delta_{j}}{\Delta_{i}} \frac{\sum_{k}^{k \leqslant \Lambda} \frac{1}{2 E_{j}(k)}}{\sum_{k}^{k \leqslant \Lambda} \frac{1}{2 E_{i}(k)}}
$$

for $i \neq j$. In the low-density region $\left(\mu<E_{0}, \Delta_{1} / E_{0} \simeq\right.$ $0, \Delta_{2} / E_{0} \simeq 0$ ), Eq. (B1) becomes

$$
\begin{gathered}
U_{i i}^{\text {eff }} \simeq U_{i i}+U_{i j} \frac{\Delta_{j}}{\Delta_{i}} \frac{\sum_{k} \frac{1}{\sum_{k} \frac{1}{k^{2} / 2 m_{j}-\mu+E_{0} \delta_{j 2}}}}{1} \\
=U_{i i}+U_{i j} \frac{\left.\Delta_{j} / 2 m_{i}-\mu+E_{0} \delta_{i 2}\right)}{\Delta_{i}} \frac{m_{j}}{m_{i}} \frac{\tilde{\Lambda}+\frac{\sqrt{\frac{m_{j}}{m_{1}}\left(\tilde{\mu}-\delta_{j 2}\right)}}{2} \ln \left|\frac{\tilde{\Lambda}-\sqrt{\frac{m_{j}}{m_{1}}\left(\tilde{\mu}-\delta_{j 2}\right)}}{\tilde{\Lambda}+\sqrt{\frac{m_{j}}{m_{1}}\left(\tilde{\mu}-\delta_{j 2}\right)}}\right|}{\tilde{\Lambda}+\frac{\sqrt{\frac{m_{i}}{m_{1}}\left(\tilde{\mu}-\delta_{i 2}\right)}}{2} \ln \left|\frac{\tilde{\Lambda}-\sqrt{\frac{m_{i}}{m_{1}}\left(\tilde{\mu}-\delta_{i 2}\right)}}{\tilde{\Lambda}+\sqrt{\frac{m_{i}}{m_{1}}\left(\tilde{\mu}-\delta_{i 2}\right)}}\right|},
\end{gathered}
$$

where we defined $\tilde{\mu} \equiv \mu / E_{0}$ and $\tilde{\Lambda} \equiv \Lambda / k_{0}$. Since we take a large cutoff such that $\sqrt{\tilde{\mu}} \ll \tilde{\Lambda}, \sqrt{\tilde{\mu}-1} \ll \tilde{\Lambda}$, we end up with

$$
U_{i i}^{\mathrm{eff}} \simeq U_{i i}+U_{i j} \frac{\Delta_{j}}{\Delta_{i}} \frac{m_{j}}{m_{i}} .
$$

Hence $U_{22}^{\text {eff }}$ depends strongly on the ratio $\Delta_{1} / \Delta_{2}$, while $U_{11}^{\text {eff }}$ depends conversely on $\Delta_{2} / \Delta_{1}$. As shown in Fig. $8, \Delta_{2} / \Delta_{1}$ for $\mu<E_{0}$ region becomes smaller as $\tilde{U}_{12}$ is decreased at a given mass ratio.
[1] The BCS-BEC Crossover and the Unitary Fermi Gas, edited by W. Zwerger, Lecture Notes in Physics Vol. 836, (Springer, Berlin, 2012).

[2] M. Randeria and E. Taylor, Crossover from Bardeen-CooperSchrieffer to Bose-Einstein condensation and the unitary Fermi gas, Annu. Rev. Condens. Matter Phys 5, 209 (2014).

[3] G. C. Strinati, P. Pieri, G. Röpke, P. Schuck, and M. Urban, The BCS-BEC crossover: From ultra-cold Fermi gases to nuclear systems, Phys. Rep. 738, 1 (2018).

[4] Y. Ohashi, H. Tajima, and P. van Wyk, BCS-BEC crossover in cold atomic and in nuclear systems, Prog. Part. Nucl. Phys. 111, 103739 (2020).

[5] H. Aoki and K. Kuroki, Superconductivity in a two-band Hubbard model, Phys. Rev. B 42, 2125 (1990).

[6] H. Aoki and K. Kuroki, Realization of Negative- $U$ Superconductivity in a Class of Purely Repulsive Systems: Interacting Carrier and Insulating Bands, Phys. Rev. Lett. 69, 3820 (1992).
[7] K. Kuroki and H. Aoki, Superconductivity in metal-insulator composite bands: A realization of negative- $U$ pairing in purely repulsive systems, Phys. Rev. B 48, 7598 (1993).

[8] K. Yamazaki, M. Ochi, D. Ogura, K. Kuroki, H. Eisaki, S. Uchida, and $\mathrm{H}$. Aoki, Superconducting mechanism for a newtype cuprate $\mathrm{Ba}_{2} \mathrm{CuO}_{3}$ based on a multiorbital Lieb lattice model, Phys. Rev. Research 2, 033356 (2020).

[9] M. V. Mazziotti, T. Jarlborg, A. Bianconi, and A. Valletta, Room temperature superconductivity dome at a Fano resonance in superlattices of wires, Europhys. Lett. 134, 17001 (2021).

[10] M. V. Mazziotti, R. Raimondi, A. Valletta, G. Campi, and A. Bianconi, Resonant multi-gap superconductivity at room temperature near a Lifshitz topological transition in sulphur hydrides, J. App. Phys. 130, 173904 (2021).

[11] T. Qian, X.-P. Wang, W.-C. Jin, P. Zhang, P. Richard, G. $\mathrm{Xu}, \mathrm{X}$. Dai, Z. Fang, J.-G. Guo, X.-L. Chen et al., Absence of a Holelike Fermi Surface for the Iron-Based $\mathrm{K}_{0.8} \mathrm{Fe}_{1.7} \mathrm{Se}_{2}$ 
Superconductor Revealed by Angle-Resolved Photoemission Spectroscopy, Phys. Rev. Lett. 106, 187001 (2011).

[12] S. Tan, Y. Zhang, M. Xia, Z. Ye, F. Chen, X. Xie, R. Peng, D. $\mathrm{Xu}, \mathrm{Q}$. Fan, H. Xu et al., Interface-induced superconductivity and strain-dependent spin density waves in $\mathrm{FeSe} / \mathrm{SrTiO}_{3}$ thin films, Nat. Mater. 12, 634 (2013).

[13] D. Liu, W. Zhang, D. Mou, J. He, Y.-B. Ou, Q.-Y. Wang, Z. Li, L. Wang, L. Zhao, S. He et al., Electronic origin of high-temperature superconductivity in single-layer FeSe superconductor, Nat. Commun. 3, 931 (2012).

[14] S. He, J. He, W. Zhang, L. Zhao, D. Liu, X. Liu, D. Mou, Y.-B. Ou, Q.-Y. Wang, Z. Li et al., Phase diagram and electronic indication of high-temperature superconductivity at $65 \mathrm{~K}$ in single-layer FeSe films, Nat. Mater. 12, 605 (2013).

[15] J. J. Lee, F. T. Schmitt, R. G. Moore, S. Johnston, Y.-T. Cui, W. Li, M. Yi, Z. K. Liu, M. Hashimoto, Y. Zhang et al., Interfacial mode coupling as the origin of the enhancement of $T_{c}$ in FeSe films on $\mathrm{SrTiO}_{3}$, Nature (London) 515, 245 (2014).

[16] H. Miao, T. Qian, X. Shi, P. Richard, T. Kim, M. Hoesch, L. Xing, X.-C. Wang, C.-Q. Jin, J.-P. Hu et al., Observation of strong electron pairing on bands without Fermi surfaces in $\mathrm{LiFe}_{1-x} \mathrm{Co}_{x} \mathrm{As}$, Nat. Commun. 6, 6056 (2015).

[17] X. H. Niu, R. Peng, H. C. Xu, Y. J. Yan, J. Jiang, D. F. Xu, T. L. Yu, Q. Song, Z. C. Huang, Y. X. Wang et al., Surface electronic structure and isotropic superconducting gap in $\left(\mathrm{Li}_{0.8} \mathrm{Fe}_{0.2}\right) \mathrm{OHFeSe}$, Phys. Rev. B 92, 060504(R) (2015).

[18] A. Charnukha, D. V. Evtushinsky, C. E. Matt, N. Xu, M. Shi, B. Büchner, N. D. Zhigadlo, B. Batlogg, and S. V. Borisenko, High-temperature superconductivity from fine-tuning of Fermi surface singularities in iron oxypnictides, Sci. Rep. 5, 18273 (2015).

[19] K. Kuroki, T. Higashida, and R. Arita, High- $T_{c}$ superconductivity due to coexisting wide and narrow bands: A fluctuation exchange study of the Hubbard ladder as a test case, Phys. Rev. B 72, 212509 (2005).

[20] X. Chen, S. Maiti, A. Linscheid, and P. J. Hirschfeld, Electron pairing in the presence of incipient bands in iron-based superconductors, Phys. Rev. B 92, 224514 (2015).

[21] A. Linscheid, S. Maiti, Y. Wang, S. Johnston, and P. J. Hirschfeld, High $T_{c}$ via Spin Fluctuations from Incipient Bands: Application to Monolayers and Intercalates of FeSe, Phys. Rev. Lett. 117, 077003 (2016).

[22] Y. Bang, Pairing mechanism of heavily electron doped FeSe systems: Dynamical tuning of the pairing cutoff energy, New J. Phys. 18, 113054 (2016).

[23] D. Kato and K. Kuroki, Many-variable variational Monte Carlo study of superconductivity in two-band Hubbard models with an incipient band, Phys. Rev. Research 2, 023156 (2020).

[24] H. Suhl, B. T. Matthias, and L. R. Walker, Bardeen-CooperSchrieffer Theory of Superconductivity in the Case of Overlaping Bands, Phys. Rev. Lett. 3, 552 (1959).

[25] J. Kondo, Superconductivity in transition metals, Prog. Theor. Phys. 29, 1 (1963).

[26] H. Aoki, Theoretical possiblities for flat band superconductivity, J. Supercond. Nov. Magn. 33, 2341 (2020).

[27] Y. Lubashevsky, E. Lahoud, K. Chashka, D. Podolsky, and A. Kanigel, Shallow pockets and very strong coupling superconductivity in $\mathrm{FeSe}_{x} \mathrm{Te}_{1-x}$, Nat. Phys. 8, 309 (2012).

[28] K. Okazaki, Y. Ito, Y. Ota, Y. Kotani, T. Shimojima, T. Kiss, S. Watanabe, C.-T. Chen, S. Niitaka, T. Hanaguri, H. Takagi,
A. Chainani, and S. Shin, Superconductivity in an electron band just above the Fermi level: possible route to BCS-BEC superconductivity, Sci. Rep. 4, 4109 (2014).

[29] S. Kasahara, T. Watashige, T. Hanaguri, Y. Kohsaka, T. Yamashita, Y. Shimoyama, Y. Mizukami, R. Endo, H. Ikeda, K. Aoyama, T. Terashima, S. Uji, T. Wolf, H. von Lhneysen, T. Shibauchi, and Y. Matsuda, Field-induced superconducting phase of FeSe in the BCS-BEC crossover, Proc. Natl. Acad. Sci. USA 111, 16309 (2014).

[30] S. Kasahara, T. Yamashita, A. Shi, R. Kobayashi, Y Shimoyama, T. Watashige, K. Ishida, T. Terashima, T. Wolf, F. Hardy, C. Meingast, H. V. Lhneysen, A. Levchenko, T. Shibauchi, and Y. Matsuda, Giant superconducting fluctuations in the compensated semimetal FeSe at the BCS-BEC crossover, Nat. Commun. 7, 12843 (2016).

[31] S. Rinott, K. B. Chashka, A. Ribak, E. D. L. Rienks, A. TalebIbrahimi, P. Le Fevre, F. Bertran, M. Randeria, and A. Kanigel, Tuning across the BCS-BEC crossover in the multiband superconductor: An angle-resolved photoemission study, Sci. Adv. 3, e1602372 (2017)

[32] Y. Nakagawa, Y. Saito, T. Nojima, K. Inumaru, S. Yamanaka, Y. Kasahara, and Y. Iwasa, Gate-controlled low carrier density superconductors: Toward the two-dimensional BCS-BEC corssover, Phys. Rev. B 98, 064512 (2018).

[33] A. Bianconi, A. Valletta, A. Perali, and N. L. Saini, Superconductivity of a striped phase at the atomic limit, Physica C: Superconductivity 296, 269 (1998).

[34] Y. Avishai, Y. B. Band, and M. Trippenbach, Feshbach Resonance without a Closed-Channel Bound State, Phys. Rev. Lett. 111, 155301 (2013).

[35] C. A. Regal, M. Greiner, and D. S. Jin, Observation of Resonance Condensation of Fermionic Atom Pairs, Phys. Rev. Lett. 92, 040403 (2004).

[36] M. W. Zwierlein, C. A. Stan, C. H. Schunck, S. M. F. Raupach, A. J. Kerman, and W. Ketterle, Condensation of Pairs of Fermionic Atoms near a Feshbach Resonance, Phys. Rev. Lett. 92, 120403 (2004).

[37] A. Schirotzek, Y.-i. Shin, C. H. Schunck, and W. Ketterle, Determination of the Superfluid Gap in Atomic Fermi Gases by Quasiparticle Spectroscopy, Phys. Rev. Lett. 101, 140403 (2008).

[38] S. Hoinka, P. Dyke, M. G. Lingham, J. J. Kinnunen, G. M. Bruun, and C. J. Vale, Goldstone mode and pair-breaking excitations in atomic Fermi superfluids, Nat. Phys. 13, 943 (2017).

[39] M. Horikoshi, M. Koashi, H. Tajima, Y. Ohashi, and M. Kuwata-Gonokami, Ground-State Thermodynamic Quantities of Homogeneous Spin-1/2 Fermions from the BCS Region to the Unitarity Limit, Phys. Rev. X 7, 041004 (2017).

[40] C. Chin, R. Grimm, P. Julienne, and E. Tiesinga, Feshbach resonances in ultracold gases, Rev. Mod. Phys. 82, 1225 (2010).

[41] R. Zhang, Y. Cheng, H. Zhai, and P. Zhang, Orbital Feshbach Resonance in Alkali-Earth Atoms, Phys. Rev. Lett. 115, 135301 (2015).

[42] J. Xu, R. Zhang, Y. Cheng, R. Qi, and H. Zhai, Reaching a Fermi-superfluid state near an orbital Feshbach resonance, Phys. Rev. A 94, 033609 (2016).

[43] L. He, J. Wang, S.-G. Peng, X.-J. Liu, and H. Hu, Strongly correlated Fermi superfluid near an orbital Feshbach resonance: Stability, equation of state, and Leggett mode, Phys. Rev. A 94 043624 (2016). 
[44] M. Iskin, Trapped ${ }^{173} \mathrm{Yb}$ Fermi gas across an orbital Feshbach resonance, Phys. Rev. A 95, 013618 (2017).

[45] S. Mondal, D. Inotani, and Y. Ohashi, Single-particle excitations and strong coupling effects in the BCS-BEC crossover regime of a rare-earth Fermi gas with an orbital Feshbach resonance, J. Phys. Soc. Jpn. 87, 084302 (2018).

[46] G. Pagano, M. Mancini, G. Cappellini, L. Livi, C. Sias, J. Catani, M. Inguscio, and L. Fallani, Strongly Interactin Gas of Two-Electron Fermions at an Orbital Feshbach Resonance, Phys. Rev. Lett. 115, 265301 (2015).

[47] M. Höfer, L. Riegger, F. Scazza, C. Hofrichter, D. R. Fernandes, M. M. Parish, J. Levinsen, I. Bloch, and S. Fölling, Observation of an Orbital Interaction-Induced Feshbach Resonance in ${ }^{173} \mathrm{Yb}$, Phys. Rev. Lett. 115, 265302 (2015).

[48] G. Cappellini, L. F. Livi, L. Franchi, D. Tusi, D. Benedicto Orenes, M. Inguscio, J. Catani, and L. Fallani, Coherent Manipuation of Orbital Feshbach Molecules of Two-Electron Atoms, Phys. Rev. X 9, 011028 (2019).

[49] A. V. Gorshkov, M. Hermele, V. Guarie, C. Xu, P. S. Julienne, J. Ye, P. Zoller, E. Demler, M. D. Lurkin, and A. M. Rey, Twoorbital SU(N) magnetism with ultracold alkaline-earth atoms, Nat. Phys. 6, 289 (2010).

[50] Y. Cheng, R. Zhang, P. Zhang, and H. Zhai, Enhancing Kondo coupling in alkaline-earth atomic gases with confinementinduced resonance in mixed dimensions, Phys. Rev. A 96, 063605 (2017).

[51] Y. Ohashi and A. Griffin, Single-particle excitations in a trapped gas of Fermi atoms in the BCS-BEC crossover region, Phys. Rev. A 72, 013601 (2005).

[52] X.-J. Liu and H. Hu, Self-consistent theory of atomic Fermi gases with a Feshbach resonance at the superfluid transition, Phys. Rev. A 72, 063613 (2005).
[53] H. Tajima, Precursor of a Superfluidity in a strongly interacting Fermi gas with negative effective range, Phys. Rev. A 97, 043613 (2018).

[54] A. J. Leggett, in Modern Trends in the Theory of Condensed Matter, edited by A. Pekalsk and J. Przystawa, (Springer, Berlin, 1980), p. 14.

[55] M. Iskin and C. A. R. Sá de Melo, Two-band superfluidity from the BCS to the BEC limit, Phys. Rev. B 74, 144517 (2006).

[56] A. Guidini and A. Perali, Band-edge BCS-BEC crossover in a two-band superconductor: physical properties and detection parameters, Supercond. Sci. Technol. 27, 124002 (2014).

[57] Y. Yerin, H. Tajima, P. Pieri, and A. Perali, Coexistence of giant Cooper pairs with a bosonic condensate and anomalous behavior of energy gaps in the BCS-BEC crossover of a two-band superfluid Fermi gas, Phys. Rev. B 100, 104528 (2019).

[58] A. V. Chubukov, I. Eremin, and D. V. Efremov, Superconductivity vs bound state formation in a two-band superconductor with small Fermi energy - applications to Fe-pnictides/chalcogenides and doped $\mathrm{SrTiO}_{3}$, Phys. Rev. B 93, 174516 (2016).

[59] L. He, H. Hu, and X.-J. Liu, Two-band description of resonant superfluidity in atomic Fermi gases, Phys. Rev. A 91, 023622 (2015).

[60] H. Tajima, Y. Yerin, A. Perali, and P. Pieri, Enhanced critical temperature, pairing fluctuation effects, and BCS-BEC crossover in a two-band Fermi gas, Phys. Rev. B 99, 180503(R) (2019).

[61] H. Tajima, Y. Yerin, P. Pieri, and A. Perali, Mechanisms of screening or enhancing the pseudogap throughout the twoband Bardeen-Cooper-Schrieffer to Bose-Einstein condensate crossover, Phys. Rev. B 102, 220504(R) (2020). 